\title{
Hélène Pelletier-Baillargeon Olivar Asselin et son temps. Le maître.
}

\author{
Montréal : Les Éditions Fides, 2010, 415 p.
}

\section{Andrée Dufour}

Centre interuniversitaire-Université Laval

Le titre de cet ouvrage pouvait suggérer un ouvrage traitant de la carrière d'enseignant d'Olivar Asselin, célèbre journaliste montréalais décédé en 1937. Or, ce troisième tome qui lui est consacré par Hélène Pelletier-Baillargeon, elle aussi journaliste et auteure bien connue, traite plus spécifiquement des divers postes de publicitaire, de journaliste et de fonctionnaire occupés par Asselin depuis les années qui suivirent sa participation à la Première Guerre mondiale jusqu'à son décès survenu en 1937. Le livre n'en contient pas moins des passages très pertinents sur l'éducation de cette période d'entre-deux-guerres qu'il convient de souligner.

Ainsi, est-il, entre autres, question dans le livre de l'École des Beaux-Arts de Montréal, de l'École des hautes études commerciales (HÉC) et de l'École polytechnique, trois institutions montréalaises prestigieuses d'enseignement supérieur. Les pages les plus intéressantes portent cependant sur " la mainmise du clergé sur l'éducation » domaine dans lequel Olivar Asselin interviendra fréquemment, en particulier dans le journal indépendant L'Ordre qu'il a fondé en 1934. L'auteure reprend ici les idées d'avant-garde du journaliste.

En effet, à une époque où le clergé assumait sans remise en question la direction du système d'enseignement du Québec, celui-ci déplore que les niveaux scolaires secondaire et collégial soient "le fief du clergé et des religieux " et que les enseignants religieux même dépourvus de diplôme soient préférés aux laïcs, un état de fait qui a pour effet de " niveler par le bas les critères de l'enseignement ». (p. 309). Dès le premier numéro de L'Ordre, il réclame la réforme de tous les ordres d'enseignement, tant l'enseignement du français et des sciences lui semble lacunaire et bien peu adapté aux besoins du temps et la pédagogie " contraire " à la psychologie de l'enfant et de l'adolescent. Il importe selon lui de faire appel à des laïcs compétents et munis de diplômes dans les matières enseignées.

Son journal ne conteste tout de même pas la confessionnalité scolaire et 
l'enseignement religieux estime Hélène Pelletier Baillargeon, la religion répondant à un " besoin spirituel fondamental " et une "voie non violente susceptible et de faire obstacle aux idées subversives et révolutionnaires qui engendrent bolchevisme fascisme et dictature ». Aux yeux des rédacteurs du journal, l'enseignement religieux catholique s'avère « le meilleur rempart contre l'idéologie protestante » et un « facteur d'ordre et de paix sociale. » (p. 310).

Le journal n'en propose pas moins des changements susceptibles d'améliorer l'enseignement, au niveau secondaire surtout. Ayant séjourné en France de 1915 à 1919, Asselin propose l'adoption du modèle du lycée français, dans lequel une place prépondérante est consacrée à l'enseignement d'un français de qualité. En guise de lecture, il avance l'idée, plutôt originale, de faire appel à la lecture quotidienne d'un extrait des journaux d'information montréalais, y compris de son journal L'Ordre.

Asselin critique aussi fortement l'enseignement universitaire marqué d'un " cléricalisme avéré » et d'un " manque de rigueur et d'exigence » dans l'embauche du personnel enseignant. Des failles qui devraient être corrigées par l'embauche de professeurs étrangers et laïcs pour mieux " secouer le conformisme affligeant » qui affecte les universités québécoises, l'Université de Montréal notamment (p. 312).

Malgré l'estime dont jouit Asselin pendant un certain temps auprès du cardinal de Québec, M ${ }^{\text {gr }}$ Camille Roy, et de ses fidèles lecteurs, l'ensemble des propos du journaliste, notamment sa revendication répétée de l'école publique et gratuite et de l'engagement de maîtres laïcs qualifiés, apparaissent comme une contestation de l'autorité religieuse et, plus précisément, du rôle traditionnel de l'Église en matière d'éducation. Lorsque La Semaine religieuse, importante publication soutenue par le haut clergé catholique, condamne ses prises de positions, L'Ordre doit cesser de paraître en 1935. Deux ans plus tard, Asselin décédait.

Dans ce livre fouillé où le lecteur peut fidèlement suivre les diverses prises de position d'Olivar Asselin, les propos du journaliste concernant notamment l'éducation sont révélateurs de la pensée laïque des intellectuels de l'entre-deux-guerres. La contestation de l'autorité cléricale en matière d'enseignement apparaît déjà au sein de la société québécoise. 